\title{
Does neoadjuvant targeted therapy provide an opportunity for resectable EGFR-mutant lung cancer: a real-world retrospective study
}

\author{
Chao $\mathrm{Lv}^{1 \#}$, Yuanyuan $\mathrm{Ma}^{1 \#}$, Qin Feng ${ }^{2}$, Fangliang $\mathrm{Lu}^{1}$, Yongkun $\mathrm{Chi}^{3}$, Nan $\mathrm{Wu}^{1}$, Jian Fang ${ }^{4}$, Yue Yang ${ }^{1}$ \\ ${ }^{1}$ Department of Thoracic Surgery II, Key Laboratory of Carcinogenesis and Translational Research (Ministry of Education), Peking University \\ Cancer Hospital \& Institute, Beijing, China; ${ }^{2}$ Department of Pathology, Key Laboratory of Carcinogenesis and Translational Research (Ministry \\ of Education), Peking University Cancer Hospital \& Institute, Beijing, China; ${ }^{3}$ Department of Radiology, Key Laboratory of Carcinogenesis and \\ Translational Research (Ministry of Education), Peking University Cancer Hospital \& Institute, Beijing, China; ${ }^{4}$ Department of Thoracic Medical \\ Oncology II, Key Laboratory of Carcinogenesis and Translational Research (Ministry of Education), Peking University Cancer Hospital \& Institute, \\ Beijing, China \\ Contributions: (I) Conception and design: C Lv, Y Ma, N Wu, Y Yang; (II) Administrative support: C Lv, Y Ma, Y Chi; (III) Provision of study \\ materials or patients: Q Feng, F Lu; (IV) Collection and assembly of data: C Lv, Y Ma, F Lu, J Fang; (V) Data analysis and interpretation: C Lv, Y \\ $\mathrm{Ma}$; (VI) Manuscript writing: All authors; (VII) Final approval of manuscript: All authors. \\ \#These authors contributed equally to this work. \\ Correspondence to: Yue Yang, MD, Department of Thoracic Surgery II, Peking University Cancer Hospital \& Institute, 52 Fucheng Road, Haidian \\ District, Beijing 100142, China. Email: zlyangyue@bjmu.edu.cn.
}

Background: Although neoadjuvant chemotherapy could improve survival outcome in resectable nonsmall cell lung cancer (NSCLC), the efficacy of neoadjuvant targeted therapy is still unclear.

Methods: We retrospectively reviewed clinical records of stage I-IIIA lung adenocarcinoma patients treated with neoadjuvant targeted therapy or chemotherapy prior to surgery. The collected data were compared between the two groups. Tumor samples were collected and analyzed by sequencing to explore the epidermal growth factor receptor-tyrosine kinase inhibitor (EGFR-TKI) resistance mechanisms.

Results: A total of 134 patients were enrolled; of these, 119 (88.8\%) had clinical stage II-IIIA disease. Radiographic response rate was significantly higher with neoadjuvant targeted therapy than with chemotherapy among patients harboring EGFR mutation [objective response rate (ORR): 55.8\% vs. 32.6\%; $\mathrm{P}=0.030$ ]. EGFR exon 19 deletion achieved better tumor response than those with exon 21 L858R mutation (ORR: $70.0 \%$ vs. $40.0 \%$; $\mathrm{P}=0.057$ ). Postoperative complications, operation time, drainage volume, and postoperative hospital length of stay were comparable between two groups. There was no difference on disease free survival (DFS) between patients receiving neoadjuvant targeted therapy and chemotherapy $(\mathrm{P}=0.871)$, but those who continued long-term adjuvant targeted therapy had significantly longer DFS than those only treated with adjuvant chemotherapy postoperatively $(\mathrm{P}=0.011)$. A series of potential molecular mechanisms of EGFR-TKI primary resistance were detected; these included BIM deletion polymorphisms, EGFR T790M mutation, and PTEN, TSC1, PIK3CA, or STAT3 mutations. Patients who presented stable disease (SD) response after TKI therapy had significantly lower EGFR mutation abundance than $\mathrm{PR}$ response $(\mathrm{P}=0.032)$.

Conclusions: Neoadjuvant EGFR-TKI appears to be more effective than conventional chemotherapy for EGFR-mutant NSCLC patients. This study provides evidence that needs to be investigated further in randomized controlled trials (RCT).

Keywords: Epidermal growth factor receptor mutation (EGFR mutation); EGFR-tyrosine kinase inhibitor (EGFR-TKI) resistance; neoadjuvant TKI; next-generation sequencing (NGS); response rate

Submitted Mar 10, 2020. Accepted for publication Jul 22, 2020.

doi: $10.21037 /$ jtd-20-1265

View this article at: http://dx.doi.org/10.21037/jtd-20-1265

(C) Journal of Thoracic Disease. All rights reserved. 


\section{Introduction}

Lung cancer is the leading cause of cancer-related death worldwide (1). In stage I-IIIA resectable disease there is a high frequency of local recurrence and distant metastasis even after complete surgical resection (2,3). Neoadjuvant chemotherapy can reduce tumor burden and eradicate micrometastasis and thus improve survival. Several randomized controlled trials (RCT) have demonstrated the survival benefit resulting from preoperative chemotherapy, with the objective response rate (ORR) ranging from $35.4 \%$ to $41 \%(4,5)$. One meta-analysis of 15 RCTs found that preoperative chemotherapy could bring about a $13 \%$ reduction in the risk of death in stage IB-IIIA resectable non-small cell lung cancer (NSCLC) patients and improve overall survival from $40 \%$ to $45 \%$ at 5 years (6).

Multiple prospective studies have shown that epidermal growth factor receptor (EGFR)-tyrosine kinase inhibitor (TKI) therapy has better efficacy and less toxicity than conventional chemotherapy for initial treatment of EGFR-mutant advanced NSCLC (7-10). However, for resectable lung cancer harboring EGFR mutation, whether preoperative targeted therapy is comparable to neoadjuvant chemotherapy is still unclear. Over last decade a few phase II studies have been conducted to evaluate the feasibility and safety of EGFR-TKI followed by surgical resection for treatment of lung cancer (11-13). All of these studies were single-armed, and most of the enrolled patients were stage I who did not harbor the EGFR mutation. Last year, a phase II randomized study (EMERGING) reported that among $72 \mathrm{~N} 2$ positive EGFR-mutant patients, the ORR for neoadjuvant erlotinib versus GC chemotherapy was $54.1 \%$ versus $34.3 \%(\mathrm{P}=0.092)$ (14).

So far, it is still not known whether preoperative EGFR-TKI should be recommended as induction strategy in EGFR-mutant patients. The optimal adjuvant treatment modality following surgery, the patient subset that would benefit from this treatment, and the mechanism of development of TKI resistance also remain to be elucidated. We conducted this real-world study to compare the efficacy and survival outcome of neoadjuvant EGFR-TKI vs. chemotherapy in patients with lung adenocarcinoma. We also examined the mechanisms underlying the primary and acquired TKI resistance by DNA sequencing of pre- and post-treatment tumor samples. We present the following article in accordance with the STROBE reporting checklist (available at http:// dx. doi. org/10. 21037/jtd-20-1265).

\section{Methods}

\section{Patients}

The patients were selected from lung adenocarcinoma cases that had received neoadjuvant chemotherapy or targeted therapy followed by surgical resection at Peking University (PKU) Cancer Hospital between October 2013 and September 2019. Patients were eligible for inclusion if (I) they had confirmed histopathological diagnosis of stage I-IIIA lung adenocarcinoma by the American Joint Committee on Cancer (AJCC) criteria, 8th edition; (II) they had received at least two cycles of platinum-based chemotherapy or at least 4 weeks of targeted therapy prior to surgery.

A thoracic surgeon and a radiologist jointly assessed radiographic response after induction therapy according to the Response Evaluation Criteria in Solid Tumors (RECIST) criteria. Demographic and clinical data (age, sex, TNM stage, disease location, preoperative treatment, radiographic response, perioperative complications, postoperative treatment, and survival) were analyzed. This study was conducted in accordance with the Declaration of Helsinki (as revised in 2013) and was approved by the institutional human research committee. All patients included in this study signed informed consent.

\section{EGFR mutation testing and next-generation sequencing (NGS)}

EGFR mutation status was tested on formalin-fixed paraffin-embedded (FFPE) biopsy samples and/or resected FFPE tumor tissue. All the analyses were performed in the Department of Pathology of PKU Cancer Hospital by TaqMan polymerase chain reaction (PCR) assay (Beijing ACCB Biotech Ltd., Beijing, China) (15).

NGS analyses were performed on frozen or FFPE tissues of both pretreatment biopsy sample and posttreatment surgically resected tumor tissue for patients who received preoperative EGFR-TKI. Genomic DNA were extracted from frozen tissue using the DNeasy Blood \& Tissue Kit (Qiagen). FFPE samples were deparaffinized with xylene and genomic DNA extraction was performed using QIAamp DNA FFPE Tissue Kit (Qiagen) according to the manufacturer's instruction. Sequencing libraries were prepared using the KAPA Hyper Prep Kit (KAPA Biosystems) with an optimized manufacturer's protocol. For target enrichment, indexed DNA libraries were pooled together for hybridization with customized biotinylated 
DNA probes (GeneseeqOne, Nanjing Geneseeq Technology Inc.) targeting 422 cancer-relevant genes (exons and selected introns for fusion detection). Enriched libraries were amplified and subjected to NGS on Illumina Hiseq platforms (Illumina) according to the manufacturer's instructions. Quality control for FASTQ file was performed by Trimmomatic. Sequencing reads were mapped to the reference sequence hg19 (Human Genome version 19) using Burrows-Wheeler Aligner (BWA-mem, v 0.7.12). VarScan 2 was employed for detection of somatic mutations. Annotation was performed using ANNOVAR (http:// annovar. openbioinformatics. org) on the hg19 reference genome and 2014 versions of standard databases and functional prediction programs. Genomic fusions and copy number variations $(\mathrm{CNVs})$ were identified by FACTERA and ADTEx (http://adtex.sourceforge.net) separately using default parameters.

\section{Statistical analysis}

SPSS version 22 (IBM Corp., Armonk, NY, USA) was used for statistical analysis. Tumor response and other variables were compared between the neoadjuvant targeted therapy group and the chemotherapy group. The chi-square test was applied for discrete variables. The independentsamples $t$-test (normal distribution) and Mann-Whitney test (abnormal distribution) was applied for continuous variables. In survival analysis, Kaplan-Meier method and the logrank test were used to compare disease-free survival (DFS) and overall survival (OS) of patients receiving preoperative targeted therapy vs. chemotherapy. Due to the impact of prognostic factors on survival results, patients with early stage and incomplete resection would be excluded from this analysis. We also excluded patients who underwent surgery last year since the follow-up period was too short and few of them recurred in the first routine surveillance after adjuvant therapy. GraphPad Prism 7.0 was used for drawing KaplanMeier curves. Statistical significance was at $\mathrm{P}<0.05$.

\section{Results}

\section{Patient characteristics}

Between 2013 and 2019, a total of 2,608 patients with lung adenocarcinoma underwent surgery by the same surgical team. Among these cases, 150 received neoadjuvant chemotherapy or targeted therapy followed by surgery in our department. Of these, 134 patients met the study criteria and were enrolled in this study (Figure 1). Pretreatment staging was evaluated by means of FDGpositron emission tomography-computed tomography (PET-CT) scan, with additional brain MRI performed to assess lymph node metastasis $(\mathrm{N} 1 / \mathrm{N} 2)$ and rule out distant metastasis (N3/M1). Endobronchial ultrasound-guided transbronchial needle aspiration (EBUS-TBNA) was performed in 19 patients and N2 disease was pathologically determined. Table 1 lists the patients' demographic and clinical characteristics.

\section{Treatment}

Of the 134 patients, 43 harboring EGFR mutation received preoperative targeted therapy. The remaining 91 received preoperative chemotherapy, of whom 43 (47.3\%) had the EGFR mutation. There was no significant difference between the neoadjuvant targeted therapy group and the chemotherapy group in mean age, disease location, clinical stage and prevalence of EGFR mutation (exon 18-21, excluding wild type). We observed more nonsmoking female received preoperative EGFR-TKI $(\mathrm{P}=0.022$ and 0.015 , respectively). For induction chemotherapy, pemetrexed combined with cisplatin was the most commonly used regimen (89.0\%); the other regimens included gemcitabine, paclitaxel, or docetaxel, combined with platinum agents. Two cycles of chemotherapy were administered to 80 patients, three cycles to 7 patients, and four cycles to 4 patients. Among those receiving targeted therapy, 21 patients received gefitinib (48.8\%), 14 received icotinib (32.6\%), 4 received afatinib, 3 received osimertinib (ChiCTR1800016948) and 1 received erlotinib. Most of patients received 4 to12 weeks' targeted therapy (93.0\%) followed by surgery. The median and mean duration of preoperative EGFR-TKI administration was 8 and 8.8 weeks, respectively.

\section{Radiographic response}

Among the 43 patients who received targeted therapy partial response (PR) was seen in 55.8\% (24/43), while increase in tumor size was seen in 2 patients. Patients with EGFR exon 19 deletion achieved better tumor response than those with exon 21 L858R mutation, although the difference was not statistically significant (ORR: 70.0\% vs. $40.0 \% ; \mathrm{P}=0.057)$. No difference in tumor response was noted between patients treated with gefitinib and with icotinib (ORR: $57.1 \%$ vs. $42.9 \%$; $\mathrm{P}=0.407$ ). There was also 


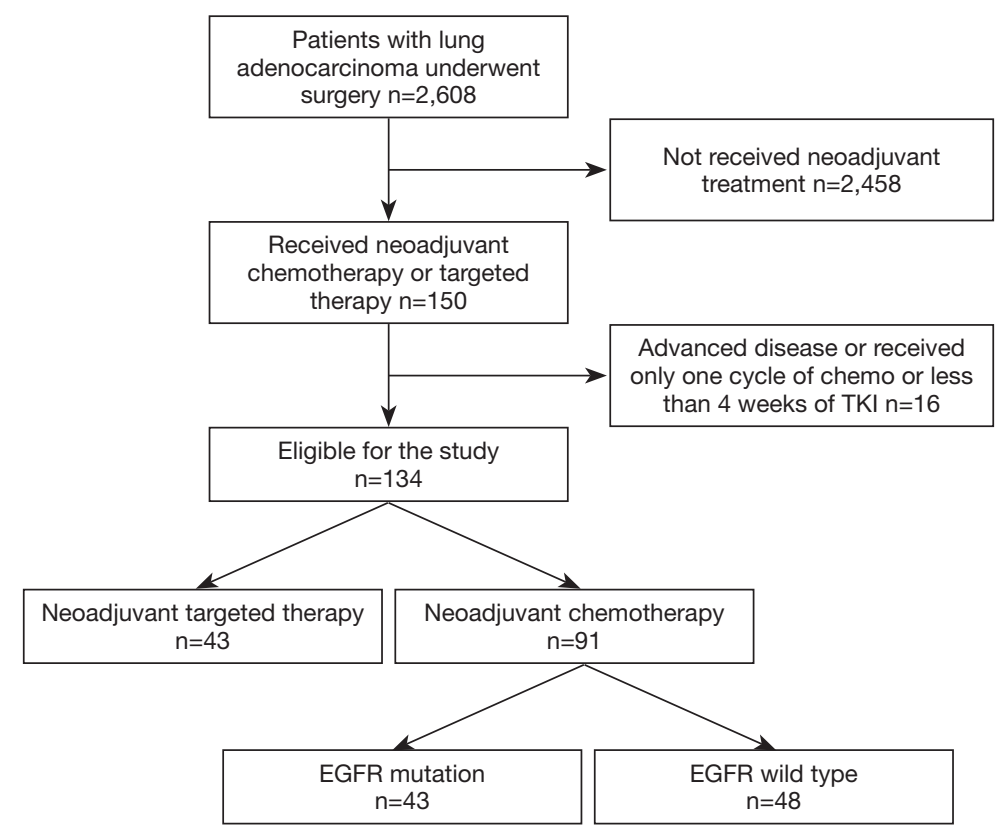

Figure 1 Flow chart of screened patients. chemo, chemotherapy; TKI, tyrosine kinase inhibitor; EGFR, epidermal growth factor receptor.

no significant correlation between different pathological subtypes of adenocarcinoma and response to EGFR-TKI $(\mathrm{P}=0.629)$. Among the 91 patients who received neoadjuvant chemotherapy, $38.5 \%$ (35/91) showed PR. Tumor response in this cohort of patients was comparable between those with and without EGFR mutation (ORR: $32.6 \%$ vs. 43.8\%; $\mathrm{P}=0.273)$. We observed that the radiographic response rate was higher with neoadjuvant targeted therapy than with chemotherapy among all the cohort of patients (ORR: $55.8 \%$ vs. $38.5 \% ; \mathrm{P}=0.059$ ), and this difference was significant in the subset of patients harboring EGFR mutation (ORR: $55.8 \%$ vs. $32.6 \%$; $\mathrm{P}=0.030$ ) (Table 1). Figure 2 shows the waterfall response plot.

\section{Surgery and survival outcome}

After neoadjuvant treatment, all patients underwent surgery (Table 2). Most patients received lobectomy with mediastinal lymph node dissection. R0 resection was performed on 127 patients (94.8\%). Of all the patients, $86(64.2 \%)$ were diagnosed as pathological stage II-III postoperatively. Two patients had pathologic complete response (pCR) after neoadjuvant chemotherapy. There were no perioperative deaths. Complication rates, operation time, amount of intraoperative bleeding, thoracic drainage volumes, duration of the drain placement, and postoperative hospital length of stay (LOS) were not significantly different between patients who received preoperative EGFR-TKI and those who received chemotherapy $(\mathrm{P}=0.688,0.625,0.720,0.895$, 0.973 , and 0.900 , respectively) (Table 2 ).

Complete follow-up data were available for 105 of the 134 patients. After excluding stage I disease ( $\mathrm{n}=15)$, R1 or R2 resection patients $(n=7)$ and those with short followup period (less than 1 year, $\mathrm{n}=28$ ), 55 cases were enrolled for survival analysis. The median follow-up duration was 33.6 months. There was no difference on disease free survival (DFS) between neoadjuvant targeted group and chemotherapy group (with and without EGFR mutation), and the median DFS was $16.7,14.1$ and 15.0 months, respectively $(\mathrm{P}=0.871$, Figure $3 A$ ). The median overall survival (OS) was not reached, and the 3 -year OS rates in TKI group and chemotherapy group were $76.6 \%$ and $66.8 \%$, respectively $(\mathrm{P}=0.719)$ (Figure $3 B)$. However, among 15 patients who received preoperative EGFR-TKI, only 8 of them continued long-term (for $>1$ year) adjuvant targeted therapy, whereas the remaining either switched to adjuvant chemotherapy or refused any adjuvant treatment after surgery (Table 3). Kaplan-Meier survival analysis revealed that preoperative TKI group patients who continued longterm adjuvant targeted therapy had significantly longer DFS than those only treated with adjuvant chemotherapy postoperatively $(\mathrm{P}=0.011)$ (Figure $3 C$ ). 
Table 1 Patients' characteristics and responses in preoperative EGFR-TKI group and chemotherapy group

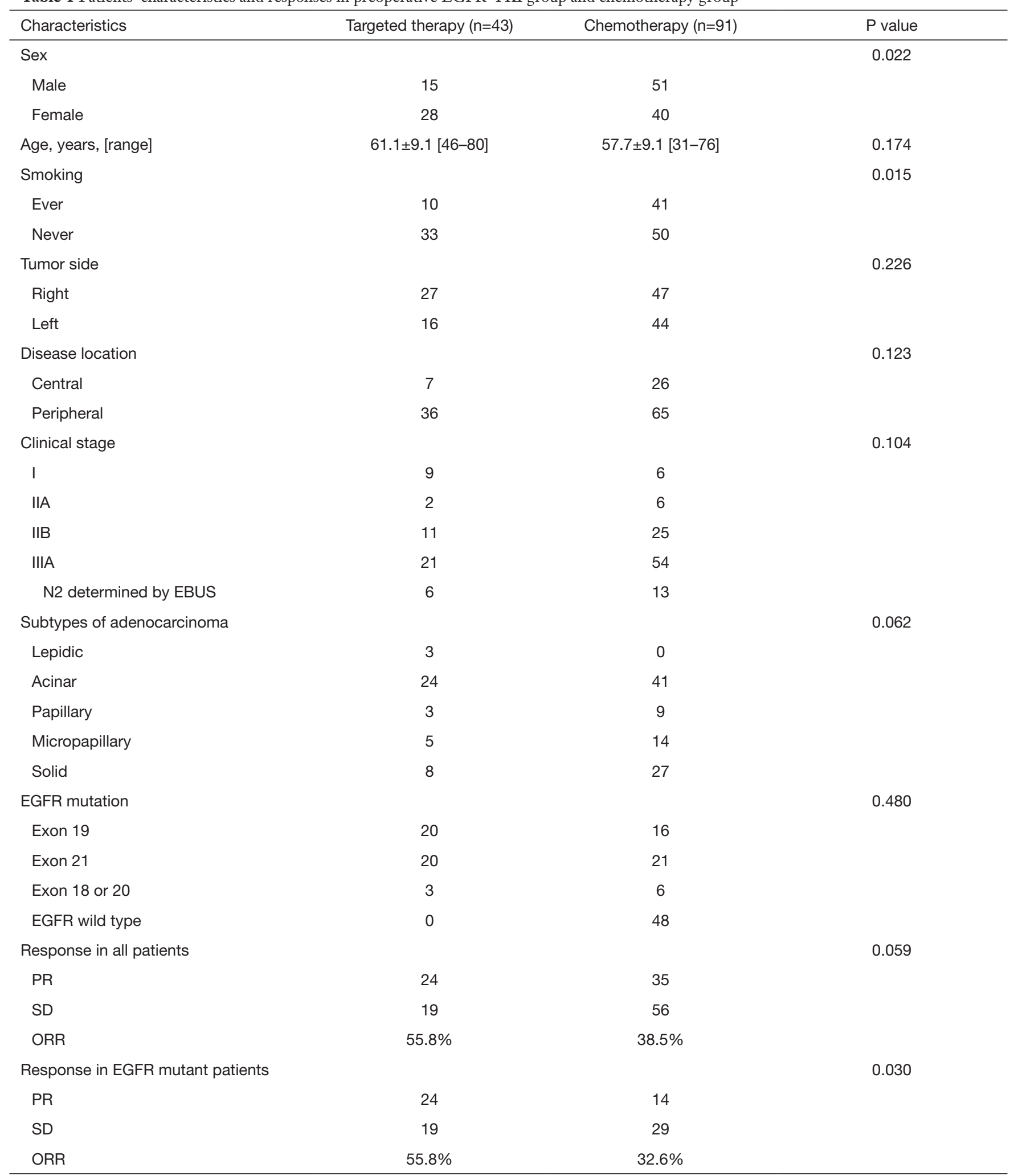

EGFR-TKI, epidermal growth factor receptor-tyrosine kinase inhibitor; EBUS, endobronchial ultrasound; PR, partial response; SD, stable disease; ORR, objective response rate. 

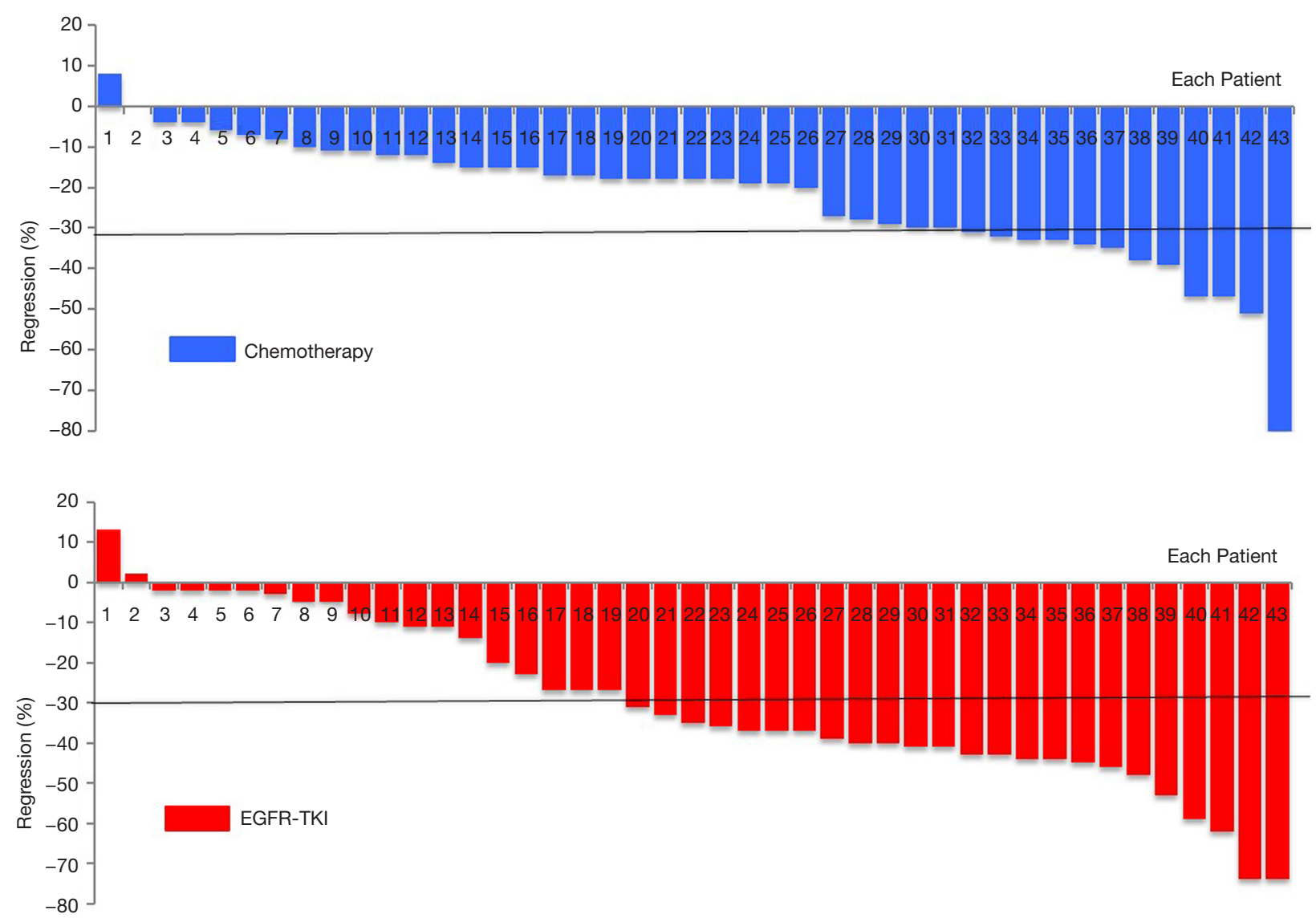

Figure 2 Waterfall response plot for patients harboring EGFR mutation; each bar shows response of individual patient. EGFR-TKI, epidermal growth factor receptor-tyrosine kinase inhibitor.

\section{Genomic profiling for molecular mechanisms for EGFR- TKI resistance}

Genomic profiling by targeted NGS of 422 cancer-relevant genes was performed on 22 surgically resected tumor samples of EGFR-positive patients treated with neoadjuvant targeted therapy; 15 matched pre-TKI tumor biopsy samples of the 22 patients were also collected and tested. The mutant allele frequency (MAF) of EGFR mutation decreased consistently after targeted therapy except in one specimen with T790M mutation (Figure 4A). Those who presented SD had significantly lower EGFR mutation MAF after TKI therapy $(\mathrm{P}=0.032$; Figure $4 B)$. In $3 \mathrm{SD}$ patients EGFR mutation was undetectable after treatment.

BIM deletion was the most common mechanism of intrinsic resistance (4/12); EGFR T790M mutation was also identified as another possible reason for occurrence of primary resistance. Mutations of other potential resistanceassociated genes, including PTEN, TSC1, PIK3CA, and
STAT3, were also detected. By analyzing paired preand post-neoadjuvant treatment samples, some adaptive mechanisms of acquired resistance were also observed, such as T790M, PTEN, NF1, AKT mutation and SCLC transformation. Figure 5 shows the detailed sequencing results (more details were seen in Table S1,S2).

\section{Discussion}

The primary aim of this study was to compare the efficacy of neoadjuvant targeted therapy with that of chemotherapy in clinical stage I-IIIA lung cancer. There are many factors favoring neoadjuvant therapy, such as attacking micrometastases at earliest time, assessing sensitivity and resistance of agents, and improving survival after surgery (16). But in clinical practice, to reduce the tumor size and improve radical resection is the major goal that all the thoracic surgeons hope to achieve, especially 
Table 2 Surgical results of all patients after neoadjuvant therapy

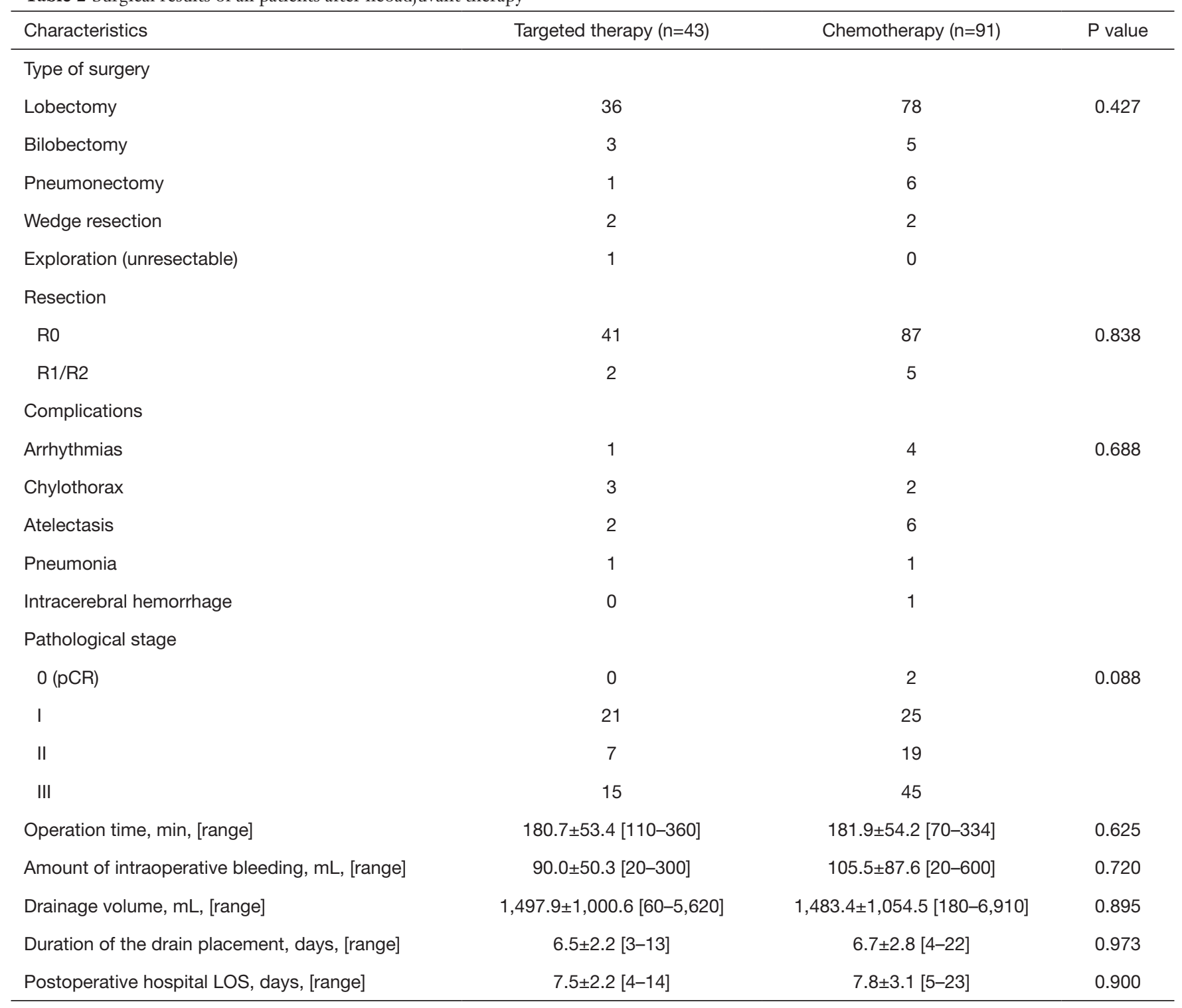

for some central diseases with great vessels invasion or with heavy tumor burden (T4). So, we compared the radiographic response rate between EGFR-TKI and conventional chemotherapy as primary assessment to evaluate the efficacy of this treatment modality. In our sample the ORR with neoadjuvant targeted therapy was $55.8 \%$ (24/43), which was consistent with EMERGING study $(54.1 \%)$, but higher than that reported in previous phase II studies enrolling EGFR-mutant patients ( $42.0 \%$ and $42.1 \%$, respectively) $(17,18)$. In the cohort of patients harboring EGFR mutation, we observed that neoadjuvant EGFR-TKI group had significantly higher ORR than chemotherapy group $(\mathrm{P}=0.030)$, but had similar postoperative complications and hospital LOS. We also noted that exon 19 deletion presented a favorable radiographic response compared with exon 21 L858R mutation (ORR: $70.0 \%$ vs. $40.0 \%$ ), which is consistent with that in advanced NSCLC patients treated with EGFR-TKI (19). These results indicate that in the clinical practice, neoadjuvant targeted therapy should be considered as a strategy of treatment for those locally advanced resectable or potentially resectable lung cancer harboring EGFR mutation, especially with exon 19 deletion, as far as ORR are concerned. 


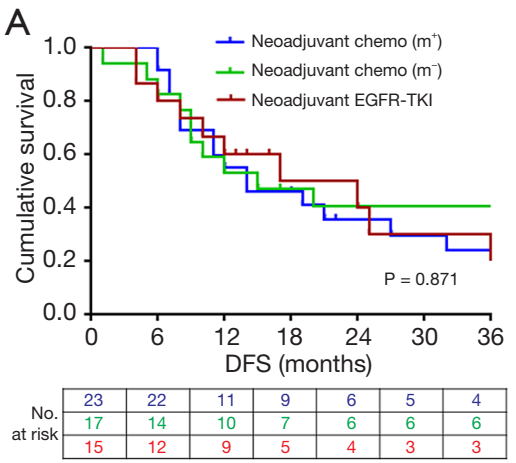

\begin{tabular}{|c|c|c|c|}
\hline & $\begin{array}{c}\text { Neoadjuvant } \\
\text { chemo }\left(\mathrm{m}^{+}\right)\end{array}$ & $\begin{array}{c}\text { Neoadjuvant } \\
\text { chemo }\left(\mathrm{m}^{-}\right)\end{array}$ & $\begin{array}{c}\text { Neoadjuvant } \\
\text { EGFR-TKI }\end{array}$ \\
\hline $\begin{array}{c}\text { Median DFS } \\
\text { (months })\end{array}$ & 14.1 & 15.0 & 15.0 \\
\hline HR $(95 \% \mathrm{Cl})$ & 1 & $\begin{array}{c}0.815 \\
(0.374-1.772)\end{array}$ & $\begin{array}{c}0.895 \\
(0.402-1.993)\end{array}$ \\
\hline
\end{tabular}

B
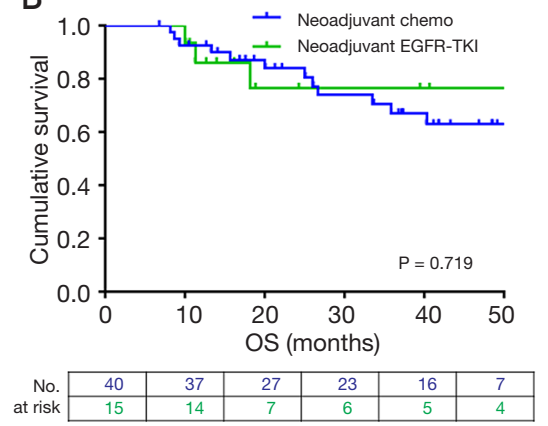

\begin{tabular}{|c|c|c|}
\hline & $\begin{array}{c}\text { Neoadjuvant } \\
\text { chemo }\end{array}$ & $\begin{array}{c}\text { Neoadjuvant } \\
\text { EGFR-TKI }\end{array}$ \\
\hline $\begin{array}{c}\text { Median DFS } \\
\text { (months) }\end{array}$ & Not reached & Not reached \\
\hline HR $(95 \% \mathrm{Cl})$ & 1 & $\begin{array}{c}0.793 \\
(0.223-2.818)\end{array}$ \\
\hline
\end{tabular}
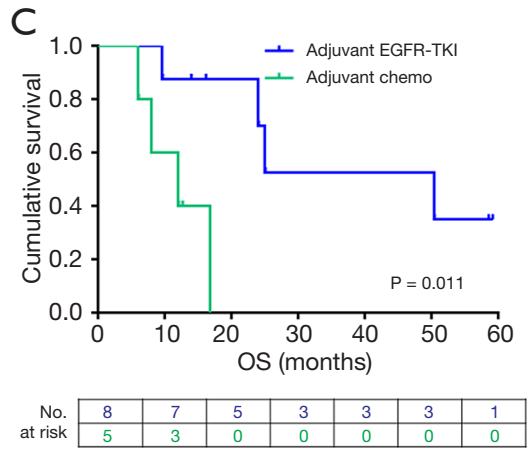

\begin{tabular}{|c|c|c|}
\hline & $\begin{array}{c}\text { Adjuvant } \\
\text { EGFR-TKI }\end{array}$ & $\begin{array}{c}\text { Adjuvant } \\
\text { chemo }\end{array}$ \\
\hline $\begin{array}{c}\text { Median DFS } \\
\text { (months ) }\end{array}$ & 50.4 & 12.0 \\
\hline HR (95\% Cl) & 1 & $\begin{array}{c}10.489 \\
(1.137-96.715)\end{array}$ \\
\hline
\end{tabular}

Figure 3 Kaplan-Meier curves of survival. (A) Disease-free survival and (B) overall survival of 55 stage II-IIIa patients receiving neoadjuvant targeted therapy or chemotherapy (with and without EGFR mutation); (C) disease-free survival of 13 patients who continued long-term adjuvant targeted therapy or only switched to adjuvant chemotherapy. chemo, chemotherapy; m, EGFR mutation; EGFR-TKI, epidermal growth factor receptor-tyrosine kinase inhibitor; DFS, disease free survival.

Table 3 Clinical data of 15 patients receiving neoadjuvant targeted therapy

\begin{tabular}{|c|c|c|c|c|c|c|c|c|}
\hline No. & Stage & Exon & TKI & Tumor size reduction (\%) & Response & Adjuvant therapy after surgery & Recurrence & DFS (mo) \\
\hline 1 & Ilb & 21 & Gefitinib & 59 & PR & No & Yes & 4.0 \\
\hline 2 & Illa & 19 & Icotinib & 41 & PR & No & Yes & 4.0 \\
\hline 3 & Ilb & 21 & Gefitinib & 27 & SD & Chemotherapy & Yes & 6.0 \\
\hline 4 & Ilb & 19 & Gefitinib & 45 & PR & Chemotherapy & Yes & 16.7 \\
\hline 5 & Ilb & 19 & Gefitinib & 53 & PR & Chemotherapy & Yes & 7.9 \\
\hline 6 & IIla & 19 & Icotinib & 48 & PR & Chemotherapy & Yes & 12.0 \\
\hline 7 & IIla & 19 & Osimertinib & 40 & PR & Chemotherapy & No & 12.6 \\
\hline 8 & Ilb & 19 & Gefitinib & 37 & PR & Gefitinib & Yes & 24.0 \\
\hline 9 & Ilb & 21 & Gefitinib & 46 & PR & Gefitinib & Yes & 9.6 \\
\hline 10 & IIla & 21 & Gefitinib & 14 & SD & Gefitinib & Yes & 25.0 \\
\hline 11 & IIla & 21 & Icotinib & 11 & SD & Chemo + Icotinib & No & 16.1 \\
\hline 12 & IIla & 18 & Icotinib & 37 & PR & Chemo + Icotinib & Yes & 50.4 \\
\hline 13 & IIla & 19 & Gefitinib & 37 & PR & Chemo + Gefitinib & No & 13.9 \\
\hline 14 & IIla & 19 & Icotinib & 39 & PR & Chemo + Icotinib & No & 59.1 \\
\hline 15 & IIla & 21 & Gefitinib & 62 & PR & Chemo + Gefitinib & No & 58.5 \\
\hline
\end{tabular}

TKI, tyrosine kinase inhibitor; PR, partial response; SD, stable disease. 
A

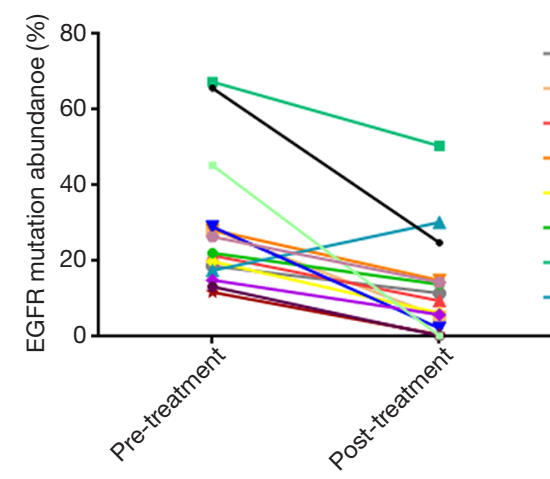

B

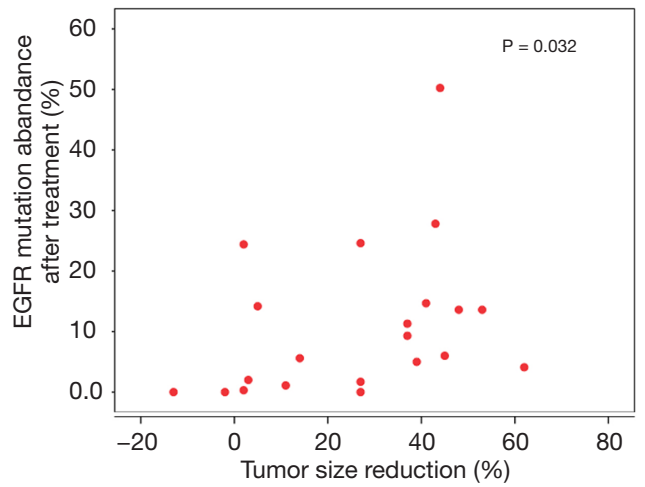

Figure 4 EGFR mutation abundance analyzed by NGS. (A) EGFR mutation abundance before and after EGFR-TKI treatment; (B) scatter plot showing correlation between tumor size reduction and EGFR mutation abundance after EGFR-TKI treatment. EGFR, epidermal growth factor receptor. NGS, next-generation sequencing; TKI, tyrosine kinase inhibitor.
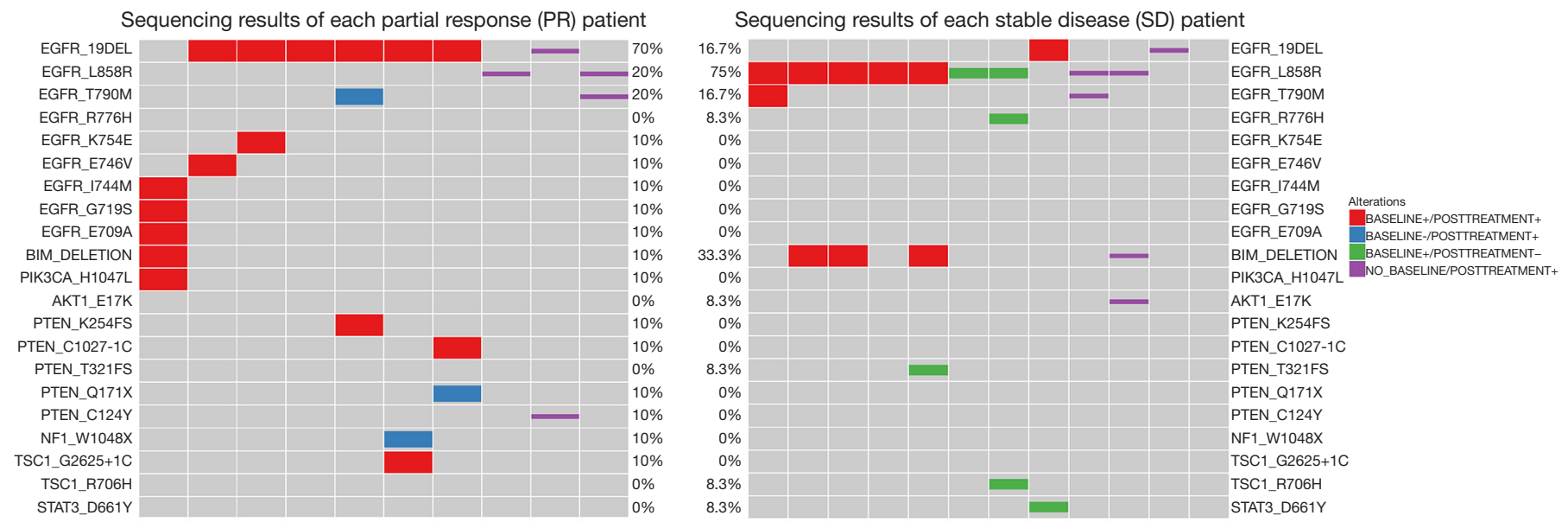

Figure 5 Sequencing results of 22 patients who received neoadjuvant targeted therapy (10 PR and 12 SD).

Besides radiographic response, the survival outcome is another crucial factor that we need to take into account to assess neoadjuvant therapy. However, in our study, the neoadjuvant targeted therapy did not prolonged DFS and OS compared with conventional chemotherapy. So far, following neoadjuvant targeted therapy and surgery, the standard adjuvant treatment modality is still unclear. Unlike perioperative chemotherapy, which is administered as three to four cycles, perioperative targeted therapy, especially adjuvant therapy, is administered continuously over a long period. Two prospective randomized studies have proved that 2 years of adjuvant targeted therapy (gefitinib or erlotinib) yields longer DFS than conventional adjuvant chemotherapy (20,21). In our study, among EGFR-mutant patients who achieved clinical response with neoadjuvant TKI therapy, those who received continued long-term adjuvant TKI therapy presented significantly longer DFS than those only treated with adjuvant chemotherapy or kept under observation. In addition, in survival analysis, of 23 EGFR-mutant patients receiving neoadjuvant chemotherapy, 7 switched to long-term adjuvant targeted therapy given that the initial tumor size reduction was minor, which might be another likely reason for the negative survival outcome on DFS. Thus, the evidence suggests that 8-12 week's preoperative EGFR-TKI, which constitutes only a small proportion of the whole duration of perioperative therapy, might not be enough to improve the survival outcome, whereas continued long-term targeted 
therapy should be administrated under the "response guidance".

Finally, if neoadjuvant targeted therapy is recommended to EGFR-mutant patients, it is necessary to identify individuals who are unsuitable for this treatment modality. Approximately 10\% of lung cancer patients have intrinsic resistance to EGFR-TKI (22), and about $30-40 \%$ patients do not present dramatic tumor response after TKI therapy, even though they harbor the EGFR mutation (7-10). Among Asian lung cancer patients $12.3 \%$ have BIM deletion polymorphism, which is one of the factors responsible for primary resistance to EGFR-TKI treatment (23). In our study, pretreatment biopsy specimens of $8 / 12$ patients assessed as SD response after preoperative TKI were sequenced and analyzed. Half of them (4/8) showed either BIM deletion polymorphism or T790M mutation, which were likely the reasons for the intrinsic resistance. In addition, 7/15 (46.7\%) patients whose pretreatment samples were analyzed had PTEN, TSC1, PIK3CA, or STAT3 mutations, which might have been the reason for poor response to EGFR-TKI or for residual tumor after TKI therapy. It has also been reported that the abundance of EGFR mutation is related to the efficacy of targeted therapy $(24,25)$. In the present study those who presented SD response had markedly lower EGFR mutation abundance or even complete absence of EGFR mutation after TKI therapy. Therefore, in addition to tests for exon 19 or 21 mutations, DNA sequencing-especially a DNA panel with a series of TKI resistance genes-could be an effective method for identifying patients in whom TKI therapy would be ineffective and thus help in avoiding unnecessary delay in surgery.

This study has some obvious limitations. It is a retrospective study from a single institution. Although PET/CT scan was performed for patients as pretreatment evaluation, not all of them underwent mediastinoscopy or EBUS-TBNA for N2 staging. The follow-up information for survival analysis in this study was inadequate and the OS was immature due to the short follow-up period and a small number of patients in each subset.

In conclusion, neoadjuvant targeted therapy appears to be more effective than conventional neoadjuvant chemotherapy in EGFR-mutant lung cancer patients, especially those with exon 19 deletion. Long-term response-guided adjuvant TKI therapy seems to be the ideal approach. DNA sequencing could be an effective method for identifying candidates of neoadjuvant TKI treatment.
Further large RCTs are necessary to clarify the effect of neoadjuvant EGFR-TKI therapy and the optimal treatment strategy.

\section{Acknowledgments}

We thank Geneseeq Technology Inc. for next generation sequencing (NGS) of all the tumor samples in this study. We are also grateful to the department of pathology in Peking University Third Hospital for their help.

Funding: This work was supported by the National Key R\&D Program of China (No. 2018YFC0910700) and National Natural Science Foundation of China (Grant number: 81772494 and 81502578 ).

\section{Footnote}

Reporting Checklist: The authors have completed the STROBE reporting checklist. Available at http://dx. doi. org/10. 21037/jtd-20-1265

Data Sharing Statement: Available at http://dx. doi. org/10. 21037/jtd-20-1265

Conflicts of Interest: All authors have completed the ICMJE uniform disclosure form (available at http://dx. doi. org/10. 21037/jtd-20-1265). The authors have no conflicts of interest to declare.

Ethics statement: The authors are accountable for all aspects of the work in ensuring that questions related to the accuracy or integrity of any part of the work are appropriately investigated and resolved. This study was conducted in accordance with the Declaration of Helsinki (as revised in 2013) and was approved by the Ethics committee of Beijing Cancer Hospital (Beijing, China). All patients included in this study signed informed consent.

Open Access Statement: This is an Open Access article distributed in accordance with the Creative Commons Attribution-NonCommercial-NoDerivs 4.0 International License (CC BY-NC-ND 4.0), which permits the noncommercial replication and distribution of the article with the strict proviso that no changes or edits are made and the original work is properly cited (including links to both the formal publication through the relevant DOI and the license). See: https://creativecommons.org/licenses/by-nc-nd/4.0/. 


\section{References}

1. Siegel RL, Miller KD, Jemal A. Cancer Statistics, 2017. CA Cancer J Clin 2017;67:7-30.

2. Goldstraw P, Crowley J, Chansky K, et al. The IASLC Lung Cancer Staging Project: proposals for the revision of the TNM stage groupings in the forthcoming (seventh) edition of the TNM classification of malignant tumors. J Thorac Oncol 2007;2:706-14.

3. Lo Cicero J III. Surgical treatment of non-small-cell lung cancer. In: Shields TW, Lo Cicero J III, Reed CE, et al. editors. General Thoracic Surgery. 7th ed. Philadelphia, PA: Lippincott Williams \& Wilkins, 2009:1387-426.

4. Scagliotti GV, Pastorino U, Vansteenkiste JF, et al. Randomized phase III study of surgery alone or surgery plus preoperative cisplatin and gemcitabine in stages IB to IIIA non-small-cell lung cancer. J Clin Oncol 2012;30:172-8.

5. Pisters KM, Vallieres E, Crowley JJ, et al. Surgery with or without preoperative paclitaxel and carboplatin in earlystage non-small-cell lung cancer: Southwest Oncology Group Trial S9900, an intergroup, randomized, phase III trial. J Clin Oncol 2010;28:1843-9.

6. NSCLC Meta-analysis Collaborative Group. Preoperative chemotherapy for none small-cell lung cancer: a systematic review and meta-analysis of individual participant data. Lancet 2014;383:1561-71.

7. Mok TS, Wu YL, Thongprasert S, et al. Gefitinib or carboplatin-paclitaxel in pulmonary adenocarcinoma. $\mathrm{N}$ Engl J Med 2009;361:947-57.

8. Maemondo M, Inoue A, Kobayashi K, et al. Gefitinib or chemotherapy for non small-cell lung cancer with mutated EGFR. N Engl J Med 2010;362:2380-8.

9. Zhou C, Wu YL, Chen G, et al. Erlotinib versus chemotherapy as first-line treatment for patients with advanced EGFR mutation-positive non-small-cell lung cancer (OPTIMAL, CTONG-0802): a multicentre, open-label, randomised, phase 3 study. Lancet Oncol 2011;12:735-42.

10. Rosell R, Carcereny E, Gervais R, et al. Erlotinib versus standard chemotherapy as first-line treatment for European patients with advanced EGFR mutation-positive non-small-cell lung cancer (EURTAC): a multicentre, open-label, randomized phase 3 trial. Lancet Oncol 2012;13:239-46.

11. Lara-Guerra H, Waddell TK, Salvarrey MA, et al. Phase II study of preoperative gefitinib in clinical stage I nonsmall-cell lung cancer. J Clin Oncol 2009;27:6229-36.
12. Rizvi NA, Rusch V, Pao W, et al. Molecular characteristics predict clinical outcomes: prospective trial correlating response to the EGFR tyrosine kinase inhibitor gefitinib with the presence of sensitizing mutations in the tyrosine binding domain of the EGFR gene. Clin Cancer Res 2011;17:3500-6.

13. Schaake EE, Kappers I, Codrington HE, et al. Tumor response and toxicity of neoadjuvant erlotinib in patients with early-stage non-small-cell lung cancer. J Clin Oncol 2012;30:2731-8.

14. Zhong WZ, Chen KN, Chen C, et al. Erlotinib Versus Gemcitabine Plus Cisplatin as Neoadjuvant Treatment of Stage IIIA-N2 EGFR-Mutant Non-Small-Cell Lung Cancer (EMERGING-CTONG 1103): A Randomized Phase II Study. J Clin Oncol 2019;37:2235-45.

15. Lv C, An C, Feng Q, et al. A retrospective study of stage I to IIIa lung adenocarcinoma after resection: what is the optimal adjuvant modality for patients with an EGFR mutation? Clin Lung Cancer 2015;16:e173-81.

16. Blumenthal GM, Bunn PA Jr, Chaft JE, et al. Current Status and Future Perspectives on Neoadjuvant Therapy in Lung Cancer. J Thorac Oncol 2018;13:1818-31.

17. Xiong L, Li R, Sun J, et al. Erlotinib as Neoadjuvant Therapy in Stage IIIA (N2) EGFR Mutation-Positive Non-Small Cell Lung Cancer: A Prospective, Single-Arm, Phase II Study. Oncologist 2019;24:157-e64.

18. Wang T, Liu Y, Zhou B, et al. Effects of icotinib on early-stage non-small-cell lung cancer as neoadjuvant treatment with different epidermal growth factor receptor phenotypes. Onco Targets Ther 2016;9:1735-41.

19. Jänne PA, Wang X, Socinski MA, et al. Randomized phase II trial of erlotinib alone or with carboplatin and paclitaxel in patients who were never or light former smokers with advanced lung adenocarcinoma: CALGB 30406 trial. J Clin Oncol 2012;30:2063-9.

20. Zhong WZ, Wang Q, Mao WM, et al. Gefitinib versus vinorelbine plus cisplatin as adjuvant treatment for stage II-IIIA (N1-N2) EGFR-mutant NSCLC (ADJUVANT/ CTONG1104): a randomised, open-label, phase 3 study. Lancet Oncol 2018;19:139-48.

21. Yue D, Xu S, Wang Q, et al. Erlotinib versus vinorelbine plus cisplatin as adjuvant therapy in Chinese patients with stage IIIA EGFR mutation-positive non-small-cell lung cancer (EVAN): a randomised, open-label, phase 2 trial. Lancet Respir Med 2018;6:863-873.

22. Takeda M, Okamoto I, Fujita Y, et al. De novo resistance to epidermal growth factor receptor-tyrosine kinase inhibitors in EGFR mutation-positive patients with non- 
small cell lung cancer. J Thorac Oncol 2010;5:399-400.

23. Ng KP, Hillmer AM, Chuah CT, et al. A common BIM deletion polymorphism mediates intrinsic resistance and inferior responses to tyrosine kinase inhibitors in cancer. Nat Med 2012;18:521-28.

24. Zhou Q, Zhang XC, Chen ZH, et al. Relative abundance of EGFR mutations predicts benefit from gefitinib

Cite this article as: $\mathrm{Lv}$ C, Ma Y, Feng Q, Lu F, Chi Y, Wu N, Fang J, Yang Y. Does neoadjuvant targeted therapy provide an opportunity for resectable EGFR-mutant lung cancer: a realworld retrospective study. J Thorac Dis 2020;12(10):5324-5335. doi: $10.21037 /$ jtd-20-1265 treatment for advanced non-small-cell lung cancer. J Clin Oncol 2011;29:3316-21.

25. Li X, Cai W, Yang G, et al. Comprehensive analysis of EGFR-mutant abundance andits effect on efficacy of EGFR-TKIs in advanced NSCLC with EGFR mutations. J Thorac Oncol 2017;12:1388-97. 
Supplementary

Table S1 Sequencing results before and after TKI treatment of each partial response (PR) patient

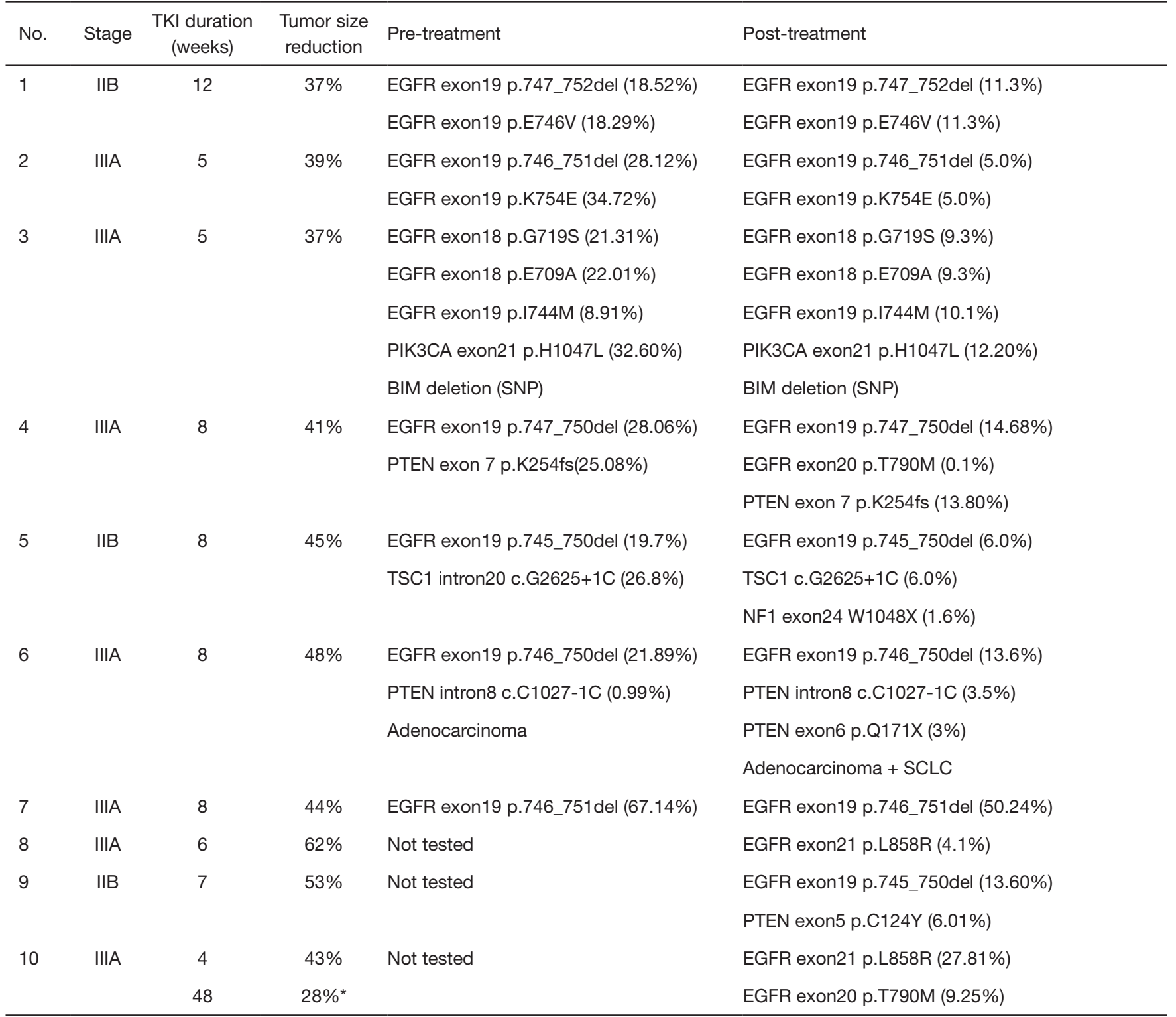

${ }^{*}$, this patient received continued TKI therapy up to 48 weeks, when tumor progressed and surgery was performed. TKI, tyrosine kinase inhibitor; EGFR, epidermal growth factor receptor; SCLC, small cell lung cancer. 
Table S2 Sequencing results before and after TKI treatment of each stable disease (SD) patient

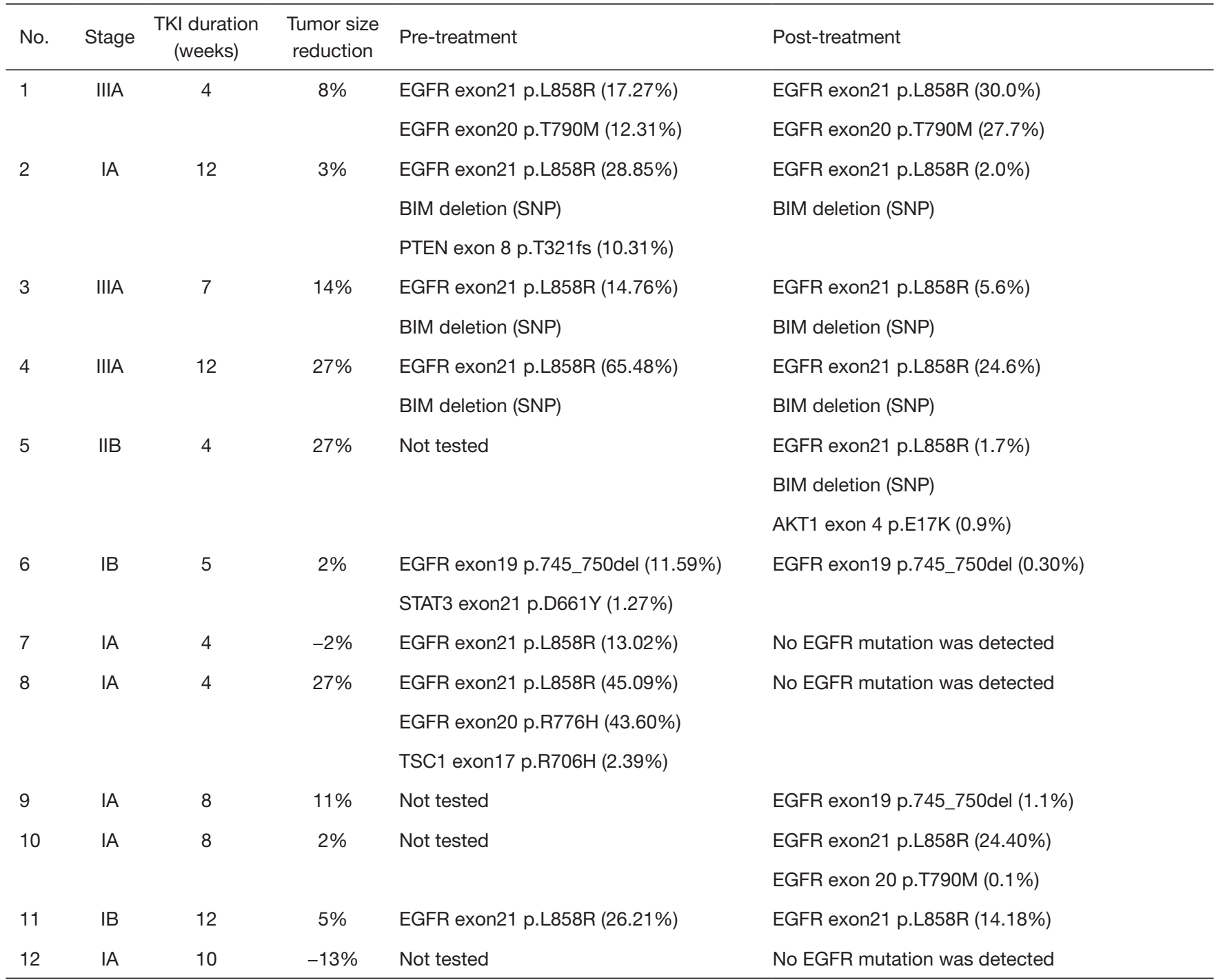

TKI, tyrosine kinase inhibitor; EGFR, epidermal growth factor receptor 